This item was submitted to Loughborough's Research Repository by the author.

Items in Figshare are protected by copyright, with all rights reserved, unless otherwise indicated.

\title{
Increasing peak capacity in nontargeted omics applications by combining full scan field asymmetric waveform ion mobility spectrometry with liquid chromatography-mass spectrometry
}

\section{PLEASE CITE THE PUBLISHED VERSION}

http://dx.doi.org/10.1021/acs.analchem.6b04315

\section{PUBLISHER}

(c) American Chemical Society

\section{VERSION}

AM (Accepted Manuscript)

\section{PUBLISHER STATEMENT}

This work is made available according to the conditions of the Creative Commons Attribution-NonCommercialNoDerivatives 4.0 International (CC BY-NC-ND 4.0) licence. Full details of this licence are available at: https://creativecommons.org/licenses/by-nc-nd/4.0/

\section{LICENCE}

CC BY-NC-ND 4.0

\section{REPOSITORY RECORD}

Arthur, Kayleigh L., Matthew Turner, Jim Reynolds, and Colin Creaser. 2017. "Increasing Peak Capacity in Nontargeted Omics Applications by Combining Full Scan Field Asymmetric Waveform Ion Mobility Spectrometry with Liquid Chromatography-mass Spectrometry". Loughborough University. https://hdl.handle.net/2134/24804. 
Increasing peak capacity in non-targeted omics applications by combining full scan field asymmetric waveform ion mobility spectrometry with liquid chromatography-mass spectrometry

Kayleigh L. Arthur, Matthew A. Turner, James C. Reynolds* and Colin S. Creaser ${ }^{\star}$ Centre for Analytical Science, Department of Chemistry, Loughborough University, Loughborough, Leicestershire, LE11 3TU, UK

Corresponding author:

Colin S Creaser (c.s.creaser@lboro.ac.uk) 


\section{Abstract (80-250 words)}

Full scan field asymmetric waveform ion mobility spectrometry (FAIMS) combined with liquid chromatography and mass spectrometry (LC-FAIMS-MS) is shown to enhance peak capacity for omics applications. A miniaturized FAIMS device capable of rapid compensation field scanning has been incorporated into an ultra-high performance liquid chromatography (UHPLC) and time-of-flight (TOF) mass spectrometry analysis, allowing the acquisition of full scan FAIMS and MS nested data sets within the timescale of a UHPLC peak. Proof of principle for the potential of scanning LC-FAIMS-MS in omics applications, is demonstrated for the non-targeted profiling of human urine using a HILIC column. The high level of orthogonality between FAIMS and MS provides additional unique compound identifiers, with detection of features based on retention time, FAIMS dispersion field and compensation field (DF and CF) and mass-to-charge $(m / z)$. Extracted FAIMS full scan data can be matched to standards to aid the identification of unknown analytes. The peak capacity for features detected in human urine using LC-FAIMS-MS was increased approximately 3-fold compared to LC-MS alone, due to a combination of the reduction of chemical noise and separation of co-eluting isobaric species across the entire analytical space. The use of FAIMS-selected in source collision induced dissociation (FISCID) yields fragmentation of ions, which reduce sample complexity associated with overlapping fragmentation patterns and provides structural information on the selected precursor ions.

\section{Keywords}

Field asymmetric waveform ion mobility spectrometry, differential ion mobility spectrometry, liquid chromatography, HILIC, mass spectrometry, omics, metabolomics, proteomics, non-targeted, collision induced dissociation 


\section{Introduction}

The identification of unknown analytes in biological matrices remains a significant challenge, whether the focus is on small molecules, metabolites, proteins, lipids or components of cellular extracts. Conventional mass spectrometric based approaches for non-targeted analyses typically utilize chromatographic techniques, such as liquid chromatography (LC) or gas chromatography, combined with mass spectrometry (MS) or tandem MS (MS/MS). ${ }^{1-3}$ However, sample matrix complexity can lead to molecular features being missed or remaining hidden within the dataset, due to components being unresolved from the chemical noise and/or co-eluting isobaric species when conventional chromatographic or mass spectrometric techniques are employed. Improvements in limits of detection and peak capacity can be achieved using complex sample preparation techniques and/or lengthy chromatographic run times. However, these approaches may not always be successful and are usually employed in targeted analyses where the analytes of interest are known. Moreover, these targeted optimization approaches may not be applicable in a non-targeted analysis, where the aim is to profile the whole metabolome or proteome and detect small perturbations within a complex matrix under specified conditions. ${ }^{4}$

Drift tube ion mobility (IM) spectrometry is a complementary technique to MS, which has been used in non-targeted IM-MS, ${ }^{5-7}$ and LC-IM-MS methods for the acquisition of nested data sets in metabolomic ${ }^{8,9}$ and proteomic applications. ${ }^{10,11}$ Field asymmetric waveform ion mobility spectrometry (FAIMS), also known as differential mobility spectrometry (DMS) or differential ion mobility spectrometry (DIMS) ${ }^{12-14}$ is an alternative to IMS which can be used to increase selectivity and sensitivity in LC-MS analysis. $^{15}$

FAIMS is an atmospheric pressure, gas-phase separation technique, which separates ions based upon non-linear compound-dependent differences in their mobilities in alternating high and low electric fields. FAIMS therefore differs from drift tube IM, which separates ions based on ion drift velocity in a buffer gas under low electric field strengths. ${ }^{14}$ In FAIMS, an asymmetric RF waveform known as the dispersion field (DF), is applied across two electrodes (planar or cylindrical), causing ions passing between the electrodes to experience a net displacement through the 
device. ${ }^{13,14}$ A superimposed DC voltage, referred to as compensation voltage (CV), is used to generate a compensation field (CF), which can be used to transmit ions of a selected differential mobility, or can be scanned to produce a FAIMS spectrum of CF versus ion intensity. FAIMS has a high degree of orthogonality with MS and LC, and FAIMS combined with LC-MS can be used to increase selectivity and lower detection limits by reducing chemical noise and removing interferences, which can be advantageous when dealing with complex matrices. ${ }^{15-17}$

FAIMS-MS has been used in non-targeted omics applications, showing increased peak capacities compared to MS alone. ${ }^{18,19}$ However, the slow scan speeds of most FAIMS devices, resulting from long ion residence and dwell times (50-200 ms), means that the acquisition of scan data over the full CF range is incompatible with typical LC peak elution times, particularly for fast ultra-high performance liquid chromatography (UHPLC) analyses. The incorporation of FAIMS into LC-MS for omics workflows has therefore been limited to stepping between a small number of selected CFs in a single run, usually between 3-6 CFs per scan, ${ }^{20,21}$ or scanning over a reduced FAIMS CF scan range; the fastest reported time for a partial CF scan being $~ 2-3$ seconds. ${ }^{22}$ Both of these approaches may result in missing data for components not transmitted through the device at the selected CFs, ${ }^{23,24}$ during the elution time of the narrow peak widths ( 5-10 s) associated with UHPLC separations. Other approaches to overcoming the slow scanning speeds of FAIMS devices have been to use long chromatographic run times to increase peak width, or to perform multiple chromatographic runs, each at a different CF, which can improve peak capacity, but significantly increases the total analysis time. ${ }^{25,26}$ There is, therefore, a need for a fast scanning of the full FAIMS CF spectrum within the timescale of a UHPLC peak, in order to acquire LC-FAIMS-MS datasets without extending the LCMS analysis time.

Fast scanning of the full CF range in a cycle time of $1 \mathrm{~s}$ is achievable using a miniaturized FAIMS device, with microsecond ion residence times, ${ }^{27-29}$ making the device compatible with UHPLC chromatographic peak widths. We report the hyphenation of UHPLC with full scan FAIMS and time-of-flight MS, for the acquisition of nested LC-FAIMS-MS data sets. The potential of this approach to omics applications is demonstrated for the molecular profiling analysis of human urine showing an increase in peak capacity without a corresponding increase in analysis 
time compared to LC-MS. A reduction in chemical noise and the separation of interferences and isobaric ions is demonstrated using a short (10 min) chromatographic method. 


\section{Experimental}

\section{Chemicals}

HPLC grade acetonitrile (ACN), water and ammonium acetate were purchased from Fisher Scientific (Loughborough, UK). Acetic acid, creatinine and theophylline were purchased from Sigma Aldrich (Dorset, UK). A pooled human urine sample was aliquoted into $500 \mu \mathrm{L}$ aliquots and stored at $-80^{\circ} \mathrm{C}$ until analyzed.

Sample preparation

A protein precipitation method previously reported was adapted and used in this study. ${ }^{9,30}$ Aliquots of urine $(500 \mu \mathrm{L})$, stored at $-80^{\circ} \mathrm{C}$, were thawed at room temperature for $20 \mathrm{mins}$. The thawed urine sample was vortexed for $30 \mathrm{~s}$ followed by ultrasonication for $1 \mathrm{~min}$ to ensure sample homogeneity, prior to the addition of cold $\left(4^{\circ} \mathrm{C}\right)$ acetonitrile $(\mathrm{ACN}, 1 \mathrm{~mL})$ to the urine aliquot. ${ }^{31}$ The mixture was vortexed for 30 $\mathrm{s}$, followed by ultrasonication for $1 \mathrm{~min}$ and allowed to stand for 5 mins prior to centrifugation at $12200 \mathrm{rpm}$ for $10 \mathrm{mins}$ at ambient temperature. The supernatant was removed and analyzed by LC-FAIMS-MS and LC-MS.

\section{LC-FAIMS-MS set-up and synchronization}

LC-FAIMS-MS analyses were performed using an Agilent 1200 series LC interfaced to an Agilent 6230 time-of-flight (TOF) MS (Agilent Technologies, Santa Clara, USA) fitted with a prototype chip-based miniaturized FAIMS device (ultraFAIMS, Owlstone Ltd., Cambridge, UK), which has been described in detail elsewhere. ${ }^{32}$ The multichannel FAIMS device (100 $\mu \mathrm{m}$ electrode gap), with a short trench length $(78.1 \mathrm{~mm})$ and ion path length $(700 \mu \mathrm{m})$, was located in front of the MS inlet transfer capillary and behind a modified spray shield in the Jet Stream electrospray (ESI) source. LCMS analyses were performed with the FAIMS device in place with no applied electric fields.

The JetStream ESI source was operated in positive ion mode in the mass range $\mathrm{m} / \mathrm{z}$ 80-1500. The MS nebulizer pressure was set to 50 psig with a sheath gas $\left(N_{2}\right)$ flow of $11 \mathrm{~L} \mathrm{~min}-1$ at $250{ }^{\circ} \mathrm{C}$ and a nozzle voltage of $2000 \mathrm{~V}$. The capillary voltage was set to $3500 \mathrm{~V}$, the fragmentor voltage was set to $200 \mathrm{~V}$ and the drying gas $\left(\mathrm{N}_{2}\right)$ flow set 
to $7 \mathrm{~L} \mathrm{~min}^{-1}$ at $150^{\circ} \mathrm{C}$. The MS scan rate was 12 scans s$^{-1}$ for LC-FAIMS-MS and LC-MS analysis, and 10 scans s $^{-1}$ for ESI-FAIMS-MS analysis.

Urine extracts $(5 \mu \mathrm{L})$ were injected onto a Poroshell 120 hydrophilic interaction liquid chromatography (HILIC) column, with dimensions $4.6 \times 50 \mathrm{~mm} \times 2.7 \mu \mathrm{m}$ (Agilent Technologies, Santa Clara, USA), maintained at $35^{\circ} \mathrm{C}$ and operated at a flow rate of $0.5 \mathrm{~mL} \mathrm{~min}{ }^{-1}$. A gradient chromatographic method utilized mobile phase A consisting of water with $10 \mathrm{mM}$ ammonium acetate and $0.1 \%$ acetic acid, and mobile phase $B$ consisting of $A C N$ water $(98: 2 \mathrm{v} / \mathrm{v})$ with $10 \mathrm{mM}$ ammonium acetate with $0.1 \%$ acetic acid. The chromatographic method was as follows: hold at $100 \% \mathrm{~B}$ from $0-0.25$ mins, decrease to $62 \%$ B from $0.25-5$ mins, increase to $100 \%$ B from 5-5.5 mins and hold for a further $4.5 \mathrm{~min}$ for re-equilibration, giving a total chromatographic run time of 10 $\min$.

The FAIMS CF was scanned from -0.9 to $4.0 \mathrm{Td}$ with DF $240 \mathrm{Td}$ at $1 \mathrm{scan} / \mathrm{s}$. Each FAIMS scan, in LC-FAIMS-MS mode, consisted of eleven 0.49 Td CF steps and an interscan re-initialization step. The FAIMS was controlled via a prototype software 'FAIMS Control' (Agilent Technologies, Santa Clara, USA) which allowed the synchronization of the FAIMS scans with TOF pushes, and was used in combination with the MassHunter Acquisition software (B.05.00, Agilent Technologies, Santa Clara, USA) which controlled the LC and the MS.

Synchronization of the start of the FAIMS scan with the start of the LC-MS acquisition was achieved via the use of a contact closure board installed on the binary LC pump (Supplementary Figure 1) connected to an in-house constructed contact closure interface, which initiated the FAIMS scanning at the start of the LC run. The contact closure interface was constructed from a computer mouse printed circuit board, which was interfaced with the computer using a USB connector and the contact closure board of the LC pump; such that, when the contact closure relay closed the left mouse button was actuated (Supplementary Figure 1). The contact closure was programmed into the chromatographic method at $0.01 \mathrm{~min}$ to trigger the start of the FAIMS scan, with the control computer mouse pointer placed over the FAIMS Control software scan execute button. This contact closure ensures that the FAIMS always began scanning at the same time point in every chromatographic run and allowed alignment and deconvolution of CF values in data analysis. 


\section{Data analysis}

The data from the LC-FAIMS-MS analysis were acquired in the mass spectrometer's output format (Agilent .d format) containing all of the data as acquired spectra labelled by retention time. The data were automatically saved in MS/MS or MS2 format ${ }^{33}$ with the FAIMS applied, and was viewed using the Agilent MassHunter Qualitative Analysis B.05.00 software (Agilent Technologies, Santa Clara, USA). The total ion chromatogram (TICs) or extracted ion chromatograms (EICs) for a selected $m / z$ at each CF were viewed in MassHunter Qualitative by extracting the data for the selected $m / z$ range at a given 'collision energy' in the extract chromatograms window, as the CF values are automatically saved in the collision energy channel using the FAIMS Control acquisition software in combination with the MassHunter Acquisition software. The resulting data sets are contained within a single file, making the exported data sets incompatible with MassHunter feature identification tools. Therefore, each individual CF dataset was exported as a separate file for further processing, using a similar approach to Canterbury et al. ${ }^{22} \mathrm{CF}$ deconvolution was achieved by extracting the mass spectra at the retention times associated with each CF (every $12^{\text {th }}$ mass spectra, including the interscan re-initialization step) and exporting the extracted spectra from MassHunter in the mzData file format, ${ }^{34}$ resulting in 11 separate files containing all of the mass spectra acquired at each CF.

The exported CF separated LC-FAIMS-MS mzData files were imported into MZmine $2.20,{ }^{34}$ an open-source software for mass spectrometry data processing and feature determination based on a signal-to-noise ratio $(\mathrm{S}: \mathrm{N})>3$. Each identified feature is a combination of retention time and $\mathrm{m} / \mathrm{z}$, creating a feature list for each CF (at the selected DF) for the LC-FAIMS-MS files. All generated feature lists were filtered to remove isotopes and adducts. The feature lists for each CF were then aligned into one list based upon retention time and $m / z$, which was exported as a csv file and opened in Microsoft Excel 2010 (Microsoft, Seattle, USA). A conditional format was applied to the aligned feature list in Excel to determine identified features in the FAIMS dimension, as features may be present in one CF or multiple CFs, which are either adjacent or separated into multiple features in the CF dimension (isobaric separation). The final feature list, with features identified by the combination of retention time, $m / z$ and CF (at a selected DF), can then be searched against online databases, such as PubChem, the human metabolome database (HMDB), the 
METLIN database and the Kyoto Encyclopedia of Genes and Genomics (KEGG) database. ${ }^{34}$

A similar approach was applied to the LC-MS data from urine and an ACN blank. The data were opened and exported from MassHunter as an mzData data file, opened in MZmine 2.20 and a feature list generated based upon a threshold $\mathrm{S}: \mathrm{N}>3$ and filtered to remove isotopes and adducts. The feature lists were exported as csv files for comparison with the LC-FAIMS-MS feature list in Microsoft Excel.

Features found from the blank ACN analysis were subtracted from the LC-MS and LC-FAIMS-MS feature lists. The remaining features were manually searched in order to ensure no chemical noise was misinterpreted as a feature. Figures presented in this report were produced using OriginLab 2015 (Academic version, b9.2.272), MassHunter Qual, and Microsoft Excel and PowerPoint. 


\section{Results and Discussion}

\section{FAIMS-MS profiling}

FAIMS-MS analysis was initially carried out on a urine extract prepared using solid phase extraction on an ODS column (see Supplementary Information). The extract was directly infused into the ESI-FAIMS-MS and a two-dimensional FAIMS sweep performed in the range DF 180-280 Td (10 Td steps), CF -2-5 Td (0.05 Td steps). A plot of DF vs. CF for the total ion count (Figure 1 (a)) shows that the majority of the ions are transmitted in the CF range -1 to $4 \mathrm{Td}$ at DFs up to $250 \mathrm{Td}$.

The high level of orthogonality between FAIMS and MS can be observed in a plot of $\mathrm{m} / \mathrm{z}$ vs CF (Figure $2(\mathrm{a})$ ) for the FAIMS-MS analysis of the directly infused urine extract. lons are observed across the entire CF region (-1 to $4 \mathrm{Td}$ ) and mass range ( $m / z$ 80-1500) showing that FAIMS can significantly increase peak capacity for omics applications. Using drift tube travelling wave ion mobility spectrometry (TWIMS), ${ }^{35}$ the corresponding plot of $m / z$ versus TWIMS drift time (bin number) shows that as the bin number increases the $m / z$ also increases (Figure $2(b)$ ), because ion collision cross section generally increases with mass. ${ }^{27}$ In comparison, FAIMS-MS shows a much weaker correlation between $\mathrm{m} / \mathrm{z}$ and CF than $\mathrm{m} / \mathrm{z}$ and bin number in TWIMSMS. The difference between FAIMS-MS and TWIMS-MS is also apparent in Supplementary Figure 2 (a) and (b). In the mass spectra extracted from the FAIMSMS data at selected CF values (Supplementary Figure 2 (a)), ions are observed across the entire $m / z$ range $(m / z 150-500)$ in each of the mass spectra as the CF increases. Whereas, in the mass spectra extracted from TWIMS-MS data at selected bin numbers (Supplementary Figure 2 (b)), the $m / z$ values observed in the spectra increase as the bin number increases. The miniaturized FAIMS device used in this study can therefore be combined with mass spectrometry to profile urine with different selectivity to IM-MS yielding characteristic DF/CF conditions which can be matched against standards to provide a unique identifier in addition to $\mathrm{m} / \mathrm{z}$. The combination of other FAIMS and DMS devices with mass spectrometry has been previously shown to increase peak capacity and resolve isobars in metabolomics and proteomic applications. ${ }^{18,36}$ 


\section{LC-FAIMS-MS profiling}

Hyphenation of liquid chromatography with mass spectrometry is widely used in omics applications, because of the orthogonal separation characteristics of the two techniques. It has also been shown that the fast scanning capability of the drift tube ion mobility spectrometry (MS timescale) allows the acquisition of nested LC-IM-MS data sets. ${ }^{8}$ In contrast, the relatively slow scan rates of most FAIMS and DMS devices means that similar datasets can only be acquired if compromises are made, such as scanning the CF over a partial range, stepping between selected CFs or using long LC run times. However, the fast scanning capability of miniaturized FAIMS ( 1s full scan), allows the acquisition of nested full scan FAIMS and MS spectra within the timescale of a UHPLC chromatographic peak width ( 5-10 s). A HILIC column was used for this study because of the widespread use of HILIC in metabolomics applications, but the approach may be extended to other separation techniques, such as reversed phase chromatography. DF $240 \mathrm{Td}$ was chosen for the LC-FAIMS-MS analysis, as a compromise between sensitivity (at low CFs) and separation of ions (at high CFs) by the FAIMS device, based on the preliminary FAIMS-MS analysis of urine (Figure 1 (a)). The FAIMS was set to scan across the entire $\mathrm{CF}$ range -0.9 to $4.0 \mathrm{Td}$, in ten $0.49 \mathrm{Td}$ steps, providing full coverage of the urine profile, with a total scan time $\sim 1 \mathrm{~s}$, which is significantly more data points/CF scan than previously reported for LC-FAIMS-MS. The total ion chromatogram for the acquired nested LC-FAIMS-MS raw data set (DF 240 Td) for urine using a HILIC UHPLC column separation is presented in Figure $3(a)$ and (b), with a single FAIMS CF scan acquired at a retention time of $7.4 \mathrm{~min}$ in Figure 3 (c). A total ion chromatogram at a selected CF $(1.55 \mathrm{Td})$ extracted from the full scan FAIMS data is shown in Figure 3 (d).

The incorporation of a FAIMS separation into the LC-TOFMS analysis adds an extra dimension of separation which is shown in Figure 1 (b) and (c). Figure 1 (b) is a plot of retention time vs. CF (at DF 240 Td) for all ions in the urine sample, where FAIMS separation of ions generated from molecules with the same retention time results in multiple peaks in the CF direction across the whole retention time range (0-10 min). Figure 1 (c) shows examples of the mass spectra at different CFs using LC-FAIMSMS (extracted from the apex of a peak in the total ion count). In this example (Figure 1 (c)), some ions can be seen in only one CF (e.g. $\mathrm{m} / z$ 400.3062, $\mathrm{m} / \mathrm{z}$ 602.2941), 
different clusters of ions can be observed at different CFs (e.g. $\mathrm{m} / z$ 380.3317452.3848, $\mathrm{m} / \mathrm{z}$ 637.5551-719.6280), and there are ions that can be observed at multiple CFs (e.g. $\mathrm{m} / \mathrm{z}$ 166.0515, $\mathrm{m} / \mathrm{z}$ 184.0608). It should be noted that ions are present across the entire mass spectral range with no apparent correlation between the increases in CF with an increase in the $\mathrm{m} / \mathrm{z}$ values observed.

The peak areas for chromatographic peaks observed at multiple CF values in the LC-FAIMS-MS data can also be used to plot extracted FAIMS CF spectral scans of chromatographic peak area vs. CF (Figure 4 (a) and (b)). These extracted FAIMS CF scans can be extremely useful in determining whether there is more than one unique FAIMS feature at a selected $m / z$ and retention time pair. For example, a peak assigned to urinary creatinine is observed in the LC-FAIMS-MS data set as the protonated and sodiated adducts at multiple CF values. The extracted FAIMS scans for these ions in urine (black) and for a creatinine standard (grey) are plotted in Figure 4 (a) and (b). From Figure 4 (a) we can observe that the protonated creatinine has one prominent feature at CF $0.08 \mathrm{Td}$, with a second weak feature present at CF $2.04 \mathrm{Td}$ in the urine, but not the standard, suggesting interference from the urine matrix. However, the sodiated creatinine has two main features, at CF $0.08 \mathrm{Td}$ and $2.04 \mathrm{Td}$, in the extracted FAIMS scan (Figure 4 (b)) for both the urinary creatinine and the standard. The two features observed indicate that the creatinine ions may be present in the gas phase in more than one form, rather than isobaric interferences from the urine matrix, because the two features are present in the standard creatinine FAIMS spectrum. Creatinine has previously been shown to coexist as two tautomers, ${ }^{37}$ and the LC-FAIMS-MS data suggest that the sodiated tautomers are separated by FAIMS. The separation of tautomers and isobars utilizing separation based on mobility is one example of the way that FAIMS can improve peak capacity in conjunction with LC-MS; two features are observed for [creatinine+Na] ${ }^{+}$using LCFAIMS-MS where only one feature is observed for [creatinine+H]+ with LC-FAIMSMS and LC-MS (Figure 4 (d)).

The extent to which LC-FAIMS-MS can increase the peak capacity in comparison to LC-MS is shown in Figure 5 (a), for the analysis of a human urine extract. A molecular feature is a defined as a unique combination of $m / z$ and retention time for LC-MS, and of $\mathrm{m} / \mathrm{z}$, retention time and CF (at DF $240 \mathrm{Td}$ ) for LC-FAIMS-MS (Figure 5 (b)). All features were filtered to remove isotopes and adducts. The number of 
molecular features observed in LC-FAIMS-MS is represented in a three-dimensional plot of CF vs. retention time vs. $\mathrm{m} / \mathrm{z}$ as shown in Figure 5 (b). The features uniquely observed in the LC-MS are plotted in Figure 5 (b) as red triangles and the features unique to LC-FAIMS-MS as blue spheres. Features common to LC-FAIMS-MS and LC-MS are plotted as purple cubes. This three-dimensional representation shows how the separation of unique features in the CF dimension is across the entire analytical space with no observable correlation between CF and either $\mathrm{m} / \mathrm{z}$ or retention time.

The total number of features found in LC-MS was 681, of which 288 were detected only using LC-MS. The total number of features found in LC-FAIMS-MS was 1838, of which 1445 were uniquely detected using LC-FAIMS-MS. Of the total features detected from both methods $68 \%$ were unique to LC-FAIMS-MS, $14 \%$ unique to LCMS and $18 \%$ common to both methods. These results clearly demonstrate that the incorporation of a FAIMS separation into LC-MS analysis significantly enhances the number of features observed. The increase in peak capacity using LC-FAIMS-MS is due to a combination of the separation of isobaric species and improvements in detection of weak ion responses in the chemical background, because of the FAIMS filtering at each CF, and hence an increase the number of features with a $S: N>3$ (Supplementary Figure 3).

It should be noted that incorporation of an ion mobility device into an LC-MS analysis usually leads to ion losses and that these losses may be greater for planar FAIMS compared to FAIMS devices with cylindrical geometry, because of the ion focusing characteristics of the latter. However, these losses are at least partially compensated by improvements in $\mathrm{S}: \mathrm{N}$ for analytes near the limit of detection. Furthermore, the incorporation of FAIMS into LC-MS does not result in the reduction in the linear dynamic range that is usually observed in drift tube IM-MS systems because of ion saturation, which can result in an extended dynamic range with FAIMS compared to MS alone. ${ }^{15}$

A total of 78 pairs of features (i.e. two features in the CF dimension at the same $\mathrm{m} / \mathrm{z}$ and retention time) were detected from the separation of isobaric species, of which 34 pairs were uniquely detected using LC-FAIMS-MS. 44 pairs were observed as one feature in the LC-MS and as two features in LC-FAIMS-MS utilizing the FAIMS 
as an extra dimension of separation. The remaining 1333 features were uniquely detected using LC-FAIMS-MS due to the reduction in chemical noise and increase in sensitivity. The peak capacity is therefore increased using the nested LC-FAIMS-MS data set without a corresponding increase in the analysis time in comparison to LCMS.

The LC-scanning FAIMS-MS non-targeted approach used a FAIMS scan rate of 1 s/scan to be compatible with UHPLC chromatographic peak widths. LC-FAIMS-MS peaks (in the deconvoluted CF chromatograms) were counted as features only if there were at least four or more data points (Supplementary Figure 4) above the baseline amplitude of the noise ( $\mathrm{S}: \mathrm{N}>3$ ), and all chromatographic peaks were visually inspected. In this study, the FAIMS was scanned from -0.9-4 Td (11 CF steps) in $1 \mathrm{~s}$. However, the ion residence time for the miniaturized FAIMS is approximately $\sim 100 \mu$ s providing (potentially) up to10,000 CF steps/s, which may be used to increase the number of data points acquired in each CF scan, the CF scan range or the number of CF scans collected across a chromatographic peak. Data acquisition using miniaturized FAIMS is therefore limited by the scan repetition rate of the TOF mass analyser, which was set to $12 \mathrm{~Hz}$ in this study, but may be increased to $30-50 \mathrm{~Hz}$ or more, depending on the maximum repetition rate of the TOF. This is in contrast to cylindrical FAIMS devices where the number of CF data points is limited by the relatively long $(50-100 \mathrm{~ms})$ ion residence time in the FAIMS device resulting in a maximum of $10 \mathrm{CF}$ steps/s for a $100 \mathrm{~ms}$ residence time.

The identification of molecular features in LC-FAIMS-MS includes the additional unique identifier of the CF (at a selected DF) in addition to retention time and $m / z$. The features observed by LC-MS and LC-FAIMS-MS using this approach can be searched against online databases using the MZmine 2 software,${ }^{34}$ by comparing $\mathrm{m} / \mathrm{z}$ and retention time data against the databases. For the identification of features dependent upon CF, such as isobaric species (multiple features at the same $\mathrm{m} / \mathrm{z}$ and retention time), standards would be required to confirm identities, as FAIMS parameters are very difficult to predict. ${ }^{38}$ The development of library data of the sort generated for LC-IM-MS would facilitate compound identification. ${ }^{39}$

An aid to the identification of molecular features is to generate fragment ions using collision induced dissociation (CID). This is possible if the FAIMS is interfaced to a 
tandem mass spectrometer, such as a Q-TOF or Q-Orbitrap. In this study, a single TOF mass analyzer was used, allowing fragment ions to be generated by in-source CID without ion pre-selection on the basis of $m / z$ and therefore the fragment ion mass spectrum at a particular retention time can be a complex array of overlapping fragmentation patterns. However, an approach previously described as FAIMS-insource collision induced dissociation-MS (FISCID-MS), ${ }^{32}$ can be used to simplify fragment ion spectra as a result of FAIMS pre-selection. Figure 6 shows the fragmentation of an ion, with $\mathrm{m} / \mathrm{z} 181.0727$ and retention time $2.53 \mathrm{~min}$, identified as protonated theophylline (a metabolite of caffeine) based on a comparison with a standard (data not shown). Using LC-MS, the identification of fragment ions in Figure 6 (black traces), at two different fragmentor voltages, is difficult as there is uncertainty as to which ions observed are derived from the pre-cursor ion rather than other higher $m / z$ ions present at the same retention time. For example, the ion at $m / z$ 105.0489 appears to be a fragment ion of protonated theophylline due to its increase in intensity at the higher fragmentor voltage (350 V). However, utilizing FAIMS preselection at CF $1.06 \mathrm{Td}$ (red traces), background ions in the mass spectra are significantly reduced allowing the identification of $\mathrm{m} / \mathrm{z} 124.0481$ as the fragment ion of the precursor at $m / z 181.0727$, which is a known fragment of theophylline. ${ }^{40}$ The absolute fragment ion intensity using FISCID has decreased in comparison to LCMS, but so has the chemical noise, so that the S:N of the fragment $\mathrm{m} / \mathrm{z} 124.0481$ (fragmentor voltage $350 \mathrm{~V}$ ) is increased by four times.

\section{Conclusion}

Scanning FAIMS analysis has been combined with UHPLC and TOFMS allowing the acquisition of nested LC-FAIMS-MS datasets for a non-targeted of omics analysis. The scan rate of the FAIMS is compatible with the peak widths associated with UHPLC separations. The approach has been demonstrated for the molecular profiling of a complex biological matrix (urine), with the peak capacity increased by approximately 3-fold compared to LC-MS. The increase in peak capacity is achieved by exploiting the orthogonality between the differential mobility separation of FAIMS and mass-to-charge separation by MS resulting in a reduction in chemical background and the separation of isobaric ions. The orthogonality between FAIMS 
and MS is also shown to be different to the orthogonality between IM and MS. The absolute signal is reduced with the by the incorporation of FAIMS, but the S:N is increases from the reduction in chemical noise, thereby enhancing the detection of low intensity ions. The FAIMS CF/DF dimension provides an additional unique molecular identifier in addition to $\mathrm{m} / \mathrm{z}$ and retention time, which can be used alone or in conjunction with fragment ion data generated by FISCID methodology to aid compound identification. The approach has been evaluated here for the molecular profiling of urine, but further work is required to validate the use of LC-FAIMS-MS for the stratification of disease states and other metabolic changes and to extend the method to other sample matrices as part of an omics workflow. This work should be of interest to those in the omics field who are looking for the opportunity to gain added information from their datasets, and could potentially be applied to omics analysis including metabolomics, lipidomics and proteomics.

\section{Acknowledgements}

The authors thank Owlstone Limited and Loughborough University for financial support, Owlstone Limited and Agilent Technologies for providing instrumentation, and Lauren Brown and Billy Boyle for helpful discussions. The authors thank Donald Jones and Liam Heaney at the Department of Cardiovascular Sciences and NHIR Leicester Cardiovascular Biomedical Research Unit (Glenfield Hospital, Leicester, UK) for the access to TWIMS-MS instrumentation. 


\section{References}

(1) Lu, W.; Bennett, B.; Rabinowitz, J. J. Chromatogr. B, 2008, 871, 236-242.

(2) Koek, M.; Jellema, R.; van der Greef, J.; Tas, A.; Hankemeier, T. Metabolomics, 2011, 7, 307-328.

(3) Aebersold, R.; Mann, M. Nature, 2003, 422, 198-207.

(4) Rochfort, S. J. Nat. Prod., 2005, 68, 1813-1820.

(5) Dwivedi, P.; Wu, P.; Klopsch, S.; Puzon, G.; Xun, L.; Hill Jr, H. Metabolomics, 2008, 4, 63-80.

(6) McLean, J.; Ruotolo, B.; Gillig, K.; Russell, D. Int. J. Mass Spectrom., 2005, 240, 301-315.

(7) Dwivedi, P.; Schultz, A.; Hill Jr., H. Int. J. Mass Spectrom., 2010, 298, 78-90.

(8) Harry, E.; Weston, D.; Bristow, A.; Wilson, I.; Creaser, C. J. Chromatogr. B, 2008, 871, 357-361.

(9) Malkar, A.; Devenport, N.; Martin, H.; Patel, P.; Turner, M.; Watson, P.; Maughan, R.; Reid, H.; Sharp, B.; Thomas, C.; Reynolds, J.; Creaser, C. Metabolomics, 2013, 9, 1192-1201.

(10) Valentine, S.; Kulchania, M.; Srebalus Barnes, C.; Clemmer, D. Int. J. Mass Spectrom., 2001, 212, 97-109.

(11) Valentine, S.; Plasencia, M.; Liu, X.; Krishnan, M.; Naylor, S.; Udseth, H.; Smith, R.; Clemmer, D. J. Proteome. Res., 2006, 5, 2977-2984.

(12) Purves, R. Anal. Bioanal. Chem., 2013, 405, 35-42.

(13) Shvartsburg, A. Differential ion mobility spectrometry: non-linear ion transport and fundamentals of FAIMS, CRC Press: Boca Raton, 2009.

(14) Eiceman, G.; Karpas, Z.; Hill Jr., H. Ion mobility spectrometry, 3rd ed.; CRC Press: Boca Raton, 2014.

(15) Smith, R.; Toutoungi, D.; Reynolds, J.; Bristow, A.; Ray, A.; Sage, A.; Wilson, I. Weston, D.; Boyle, B.; Creaser, C. J. Chromatogr. A, 2013, 1278, 76-81.

(16) Kapron, J.; Jemal, M.; Duncan, G.; Kolakowski, B.; Purves, R. Rapid Commun. Mass Spectrom., 2005, 19, 1979-1983. 
(17) Da Costa, C.; Turner, M.; Reynolds, J.; Whitmarsh, S.; Lynch, T.; Creaser, C. Anal. Chem., 2016, 88, 2453-2458.

(18) Beach, D.; Gabryelski, W. Anal. Chem., 2011, 83, 9107-9113.

(19) Beach, D.; Gabryelski, W. Anal. Chem., 2013, 85, 2127-2134.

(20) Venne, K.; Bonneil, E., Eng, K.; Thibault, P. Anal Chem, 2005, 77, 2176-2186.

(21) Creese, A.; Shimwell, N.; Larkins, K.; Heath, J.; Cooper, H. J Am Soc Mass Spectrom, 2013, 24, 431-443.

(22) Canterbury, J.; Yi, X.; Hoopmann, M.; MacCoss, M. Anal Chem, 2008, 80, 68886897.

(23) Bridon, G.; Bonneil, E.; Muratore-Schroeder, T.; Caron-Lizotte, O.; Thibault, P. Journal of Proteome Research, 2012, 11, 927-940.

(24) Saba, J.; Bonneil, E.; Pomies, C.; Eng, K.; Thibault, P. Journal of Proteome Research, 2009, 8, 3355-3366.

(25) Creese, A.; Smart, J.; Cooper, H. Anal. Chem., 2013, 85, 4836-4843.

(26) Swearingen, K.; Hoopmann, M.; Johnson, R.; Saleem, R.; Aitchison, J.; Moritz, R. Mol. Cell. Proteomics, 2012, 11, M111.014985.

(27) Shvartsburg, A.; Tang, K.; Smith, R.; Holden, M.; Rush, M.; Thompson, A.; Toutoungi, D. Anal. Chem., 2009, 81, 8048-8053.

(28) Shvartsburg, A.; Smith, R.; Wilks, A.; Koehl, A.; Ruiz-Alonso, D.; Boyle, B. Anal. Chem., 2009, 81, 6489-6495.

(29) Wilks, A.; Hart, M.; Koehl, A.; Somerville, J.; Boyle, B.; Ruiz-Alonso, D. Int. J. Ion Mobil. Spec., 2012, 15, 199-222.

(30) Malkar, A.; Wilson, E.; Harrison, T.; Shaw, D.; Creaser, C. Anal. Methods, 2016, 8, 5407-5413.

(31) Polson, C.; Sarkar, P.; Incledon, B.; Raguvaran, V.; Grant, R. J Chromatogra B, 2003, 785, 263-275.

(32) Brown, L.; Smith, R.; Toutoungi, D.; Reynolds, J.; Bristow, A.; Ray, A.; Sage, A.; Wilson, I.; Weston, D.; Boyle, B.; Creaser, C. Anal Chem, 2012, 84, 4095-4103.

(33) McDonald, W.; Tabb, D.; Sadygov, R.; MacCoss, M.; Venable, J.; Graumann, J.; Johnson, J.; Cociorva, D.; Yates III, J. Rapid Commum. Mass Spectrom., 2004, 
$18,2162-2168$.

(34) Pluskal, T.; Castillo, S.; Villar-Briones, A.; Orešič, M. BMC Bioinformatics, 2010, $11,395$.

(35) Shvartsburg, A.; Smith, R. Anal. Chem., 2008, 80, 9689-9699.

(36) Martínez-Lozano, P.; Criado, E.; Vidal, G.; Cristoni, S.; Franzoso, F.; Piatti, M.; Brambilla, P. Metabolomics, 2013, 9, S30-S43.

(37) Gao, J.; Hu, Y.; Li, S.; Zhang, Y.; Chen, X. Chemical Physics, 2013, 410, 81-89.

(38) Prasad, S.; Tang, K.; Manura, D.; Papanastasiou, D.; Smith, R. Anal. Chem., 2009, 81, 8749-8757.

(39) Paglia, G.; Williams, J.; Menikarachchi, L.; Thompson, J.; Tyldesley-Worster, R.; Halldórsson, S.; Rolfsson, O.; Moseley, A.; Grant, D.; Langridge, J.; Palsson, B.; Astarita, G. Anal. Chem., 2014, 86, 3985-3993.

(40) Zhang, Y.; Mehrota, N.; Budha, N.; Christensen, M.; Meibohm, B. Clinica Chimica Acta, 2008, 398, 105-112. 


\section{Figures}

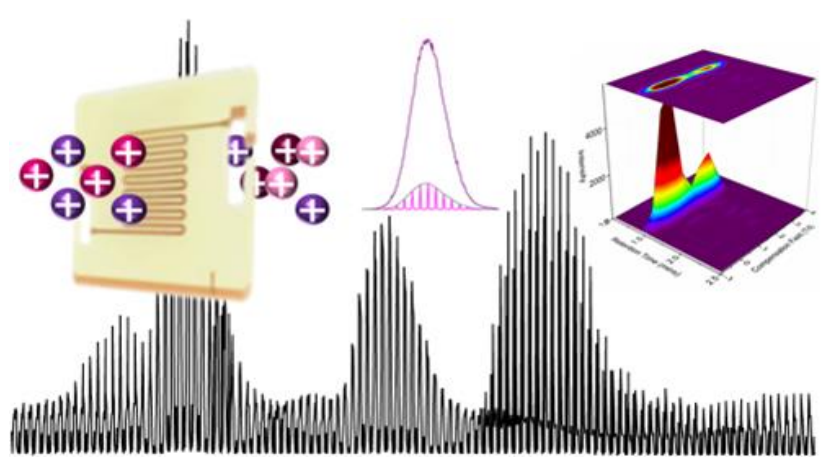

Graphical abstract figure
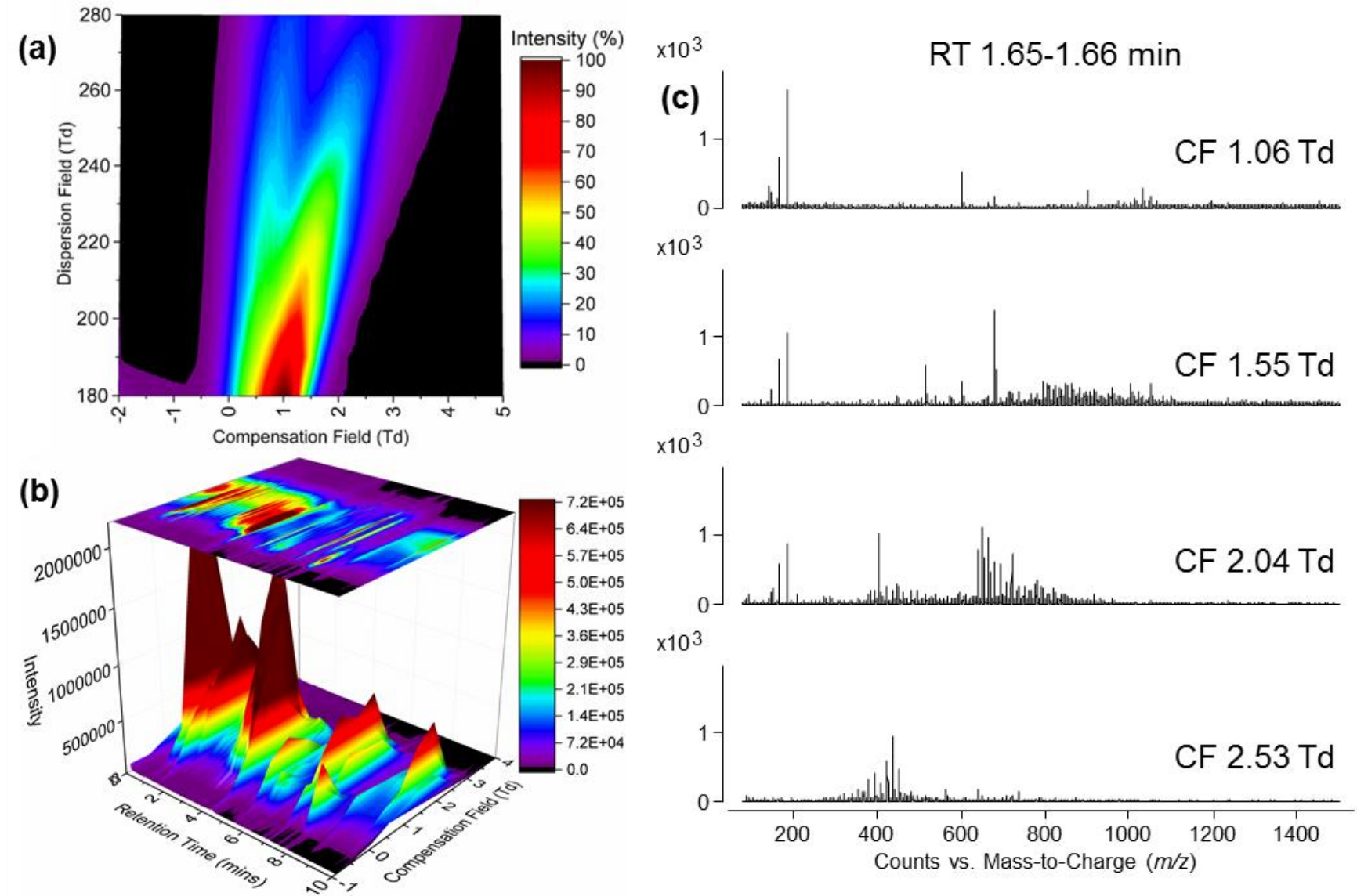

Figure 1: ESI-FAIMS-MS analysis of a urine extract, (a) Direct infusion ESI-FAIMS-MS heat plot of DF vs CF (with percentage intensity on the color scale); (b) three-dimensional plot, with two-dimensional projection, of retention time vs CF for LC-FAIMS-MS; (c) mass spectra extracted from LC-FAIMS-MS dataset at the same retention time and four different CF values. 

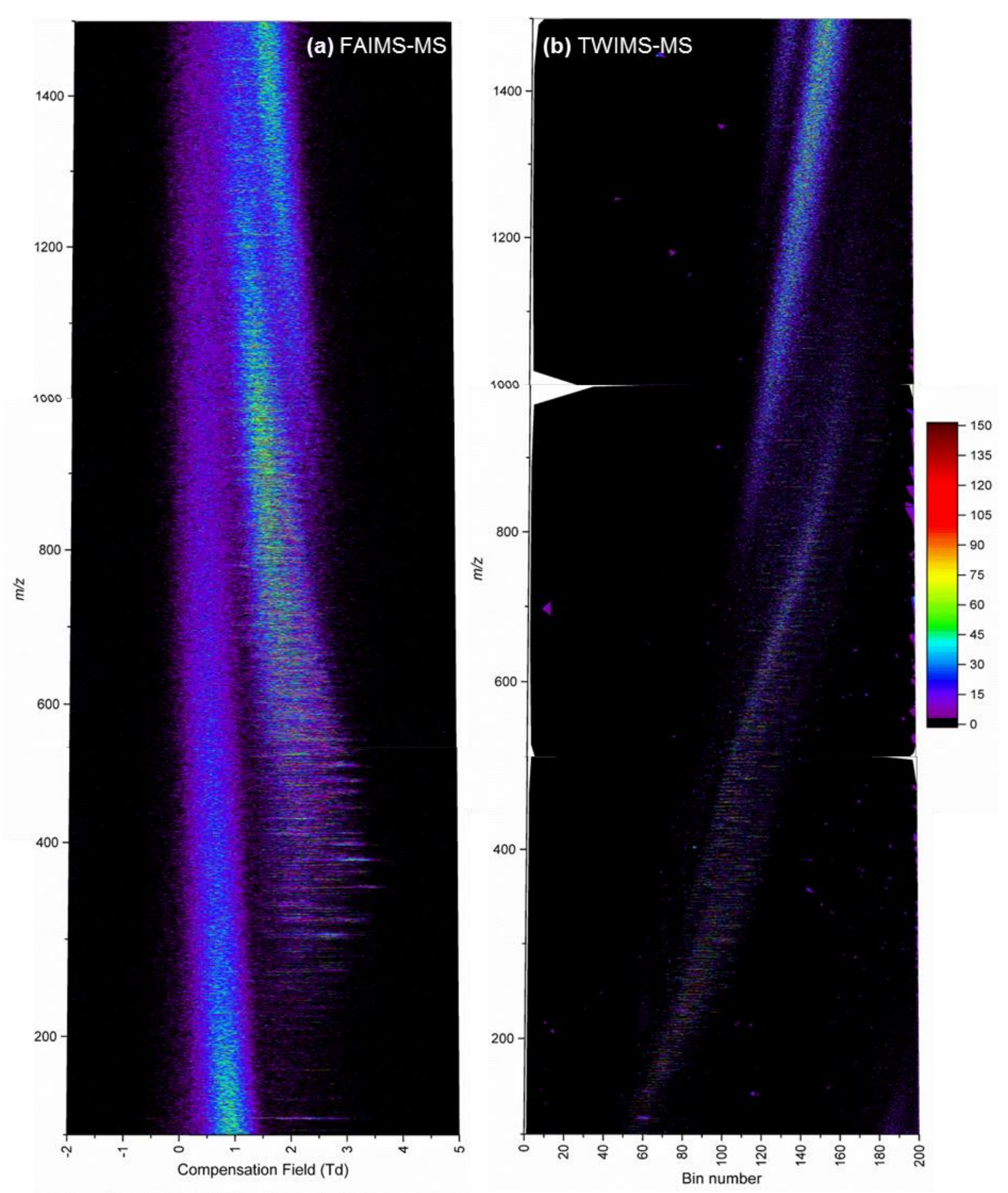

Figure 2: Heat plots for the direct infusion of urine (post SPE sample pre-treatment) using (a) ESI-FAIMS-MS ( $m / z$ vs. CF) and (b) ESI-TWIMS-MS ( $m / z$ vs. bin number). 

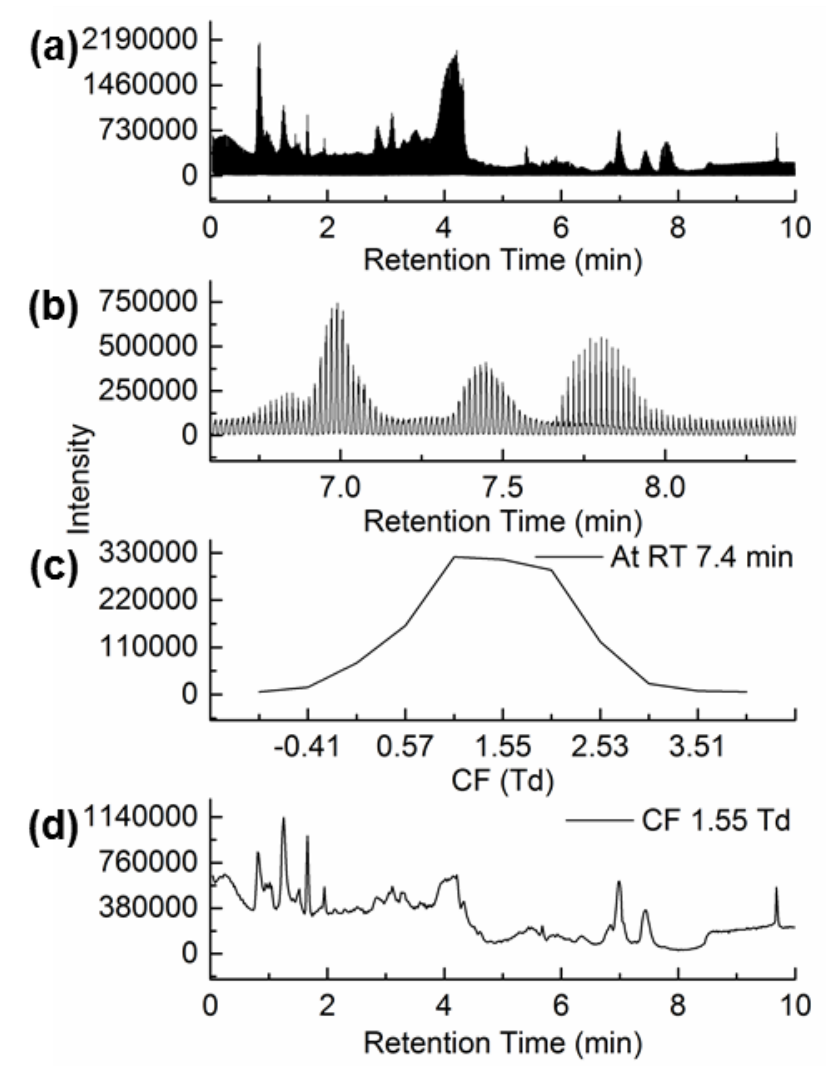

Figure 3: The LC-FAIMS-MS analysis of human urine (DF $240 \mathrm{Td}$ ), (a) total ion chromatogram (TIC) (b) zoomed in the region 6.6-8.4 min, (c) a single FAIMS scan acquired at a retention time of $7.4 \mathrm{~min}$, (d) FAIMS-selected extracted TIC chromatogram at CF 1.55 Td. 

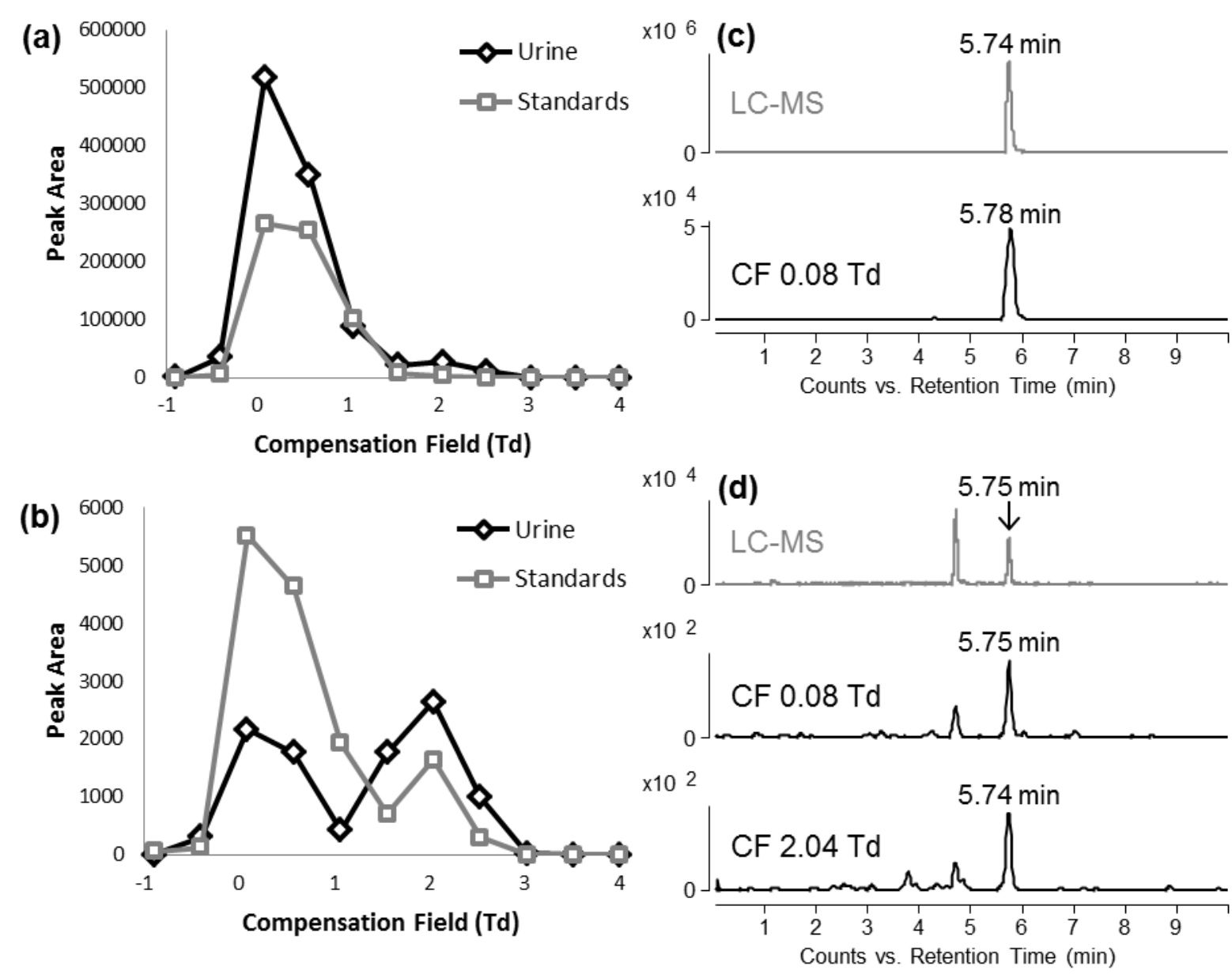

Figure 4: FAIMS scans extracted from LC-FAIMS-MS (DF $240 \mathrm{Td}$ ) of creatinine in urine (black) and a creatinine standard (grey) for (a) [creatinine $+\mathrm{H}]^{+}(\mathrm{m} / \mathrm{z} 114.0662)$ and (b)

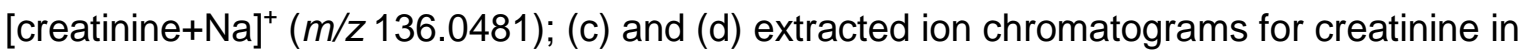
urine using LC-MS (grey) and at max CF for transmission of each FAIMS peak (black) for (c)

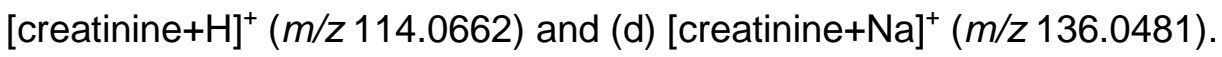




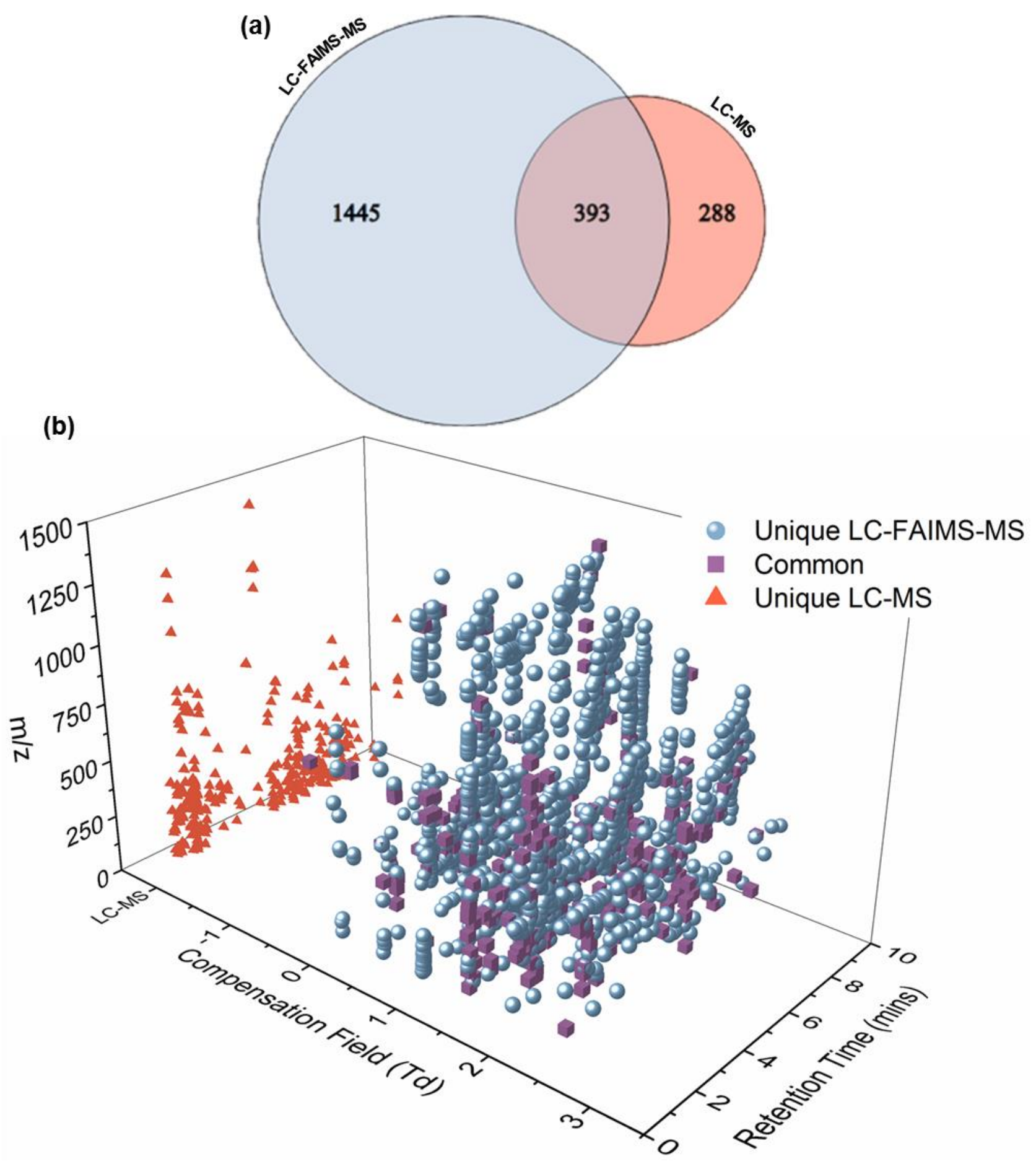

Figure 5: (a) The number of molecular features observed in LC-FAIMS-MS (blue) and LCMS (red), and the features common to both methods (purple) as a Venn diagram; (b) a three-dimensional representation of the features observed in LC-FAIMS-MS with unique features observed in LC-FAIMS-MS (blue spheres) and LC-MS (red triangles), and the features common to both methods (purple cubes). 


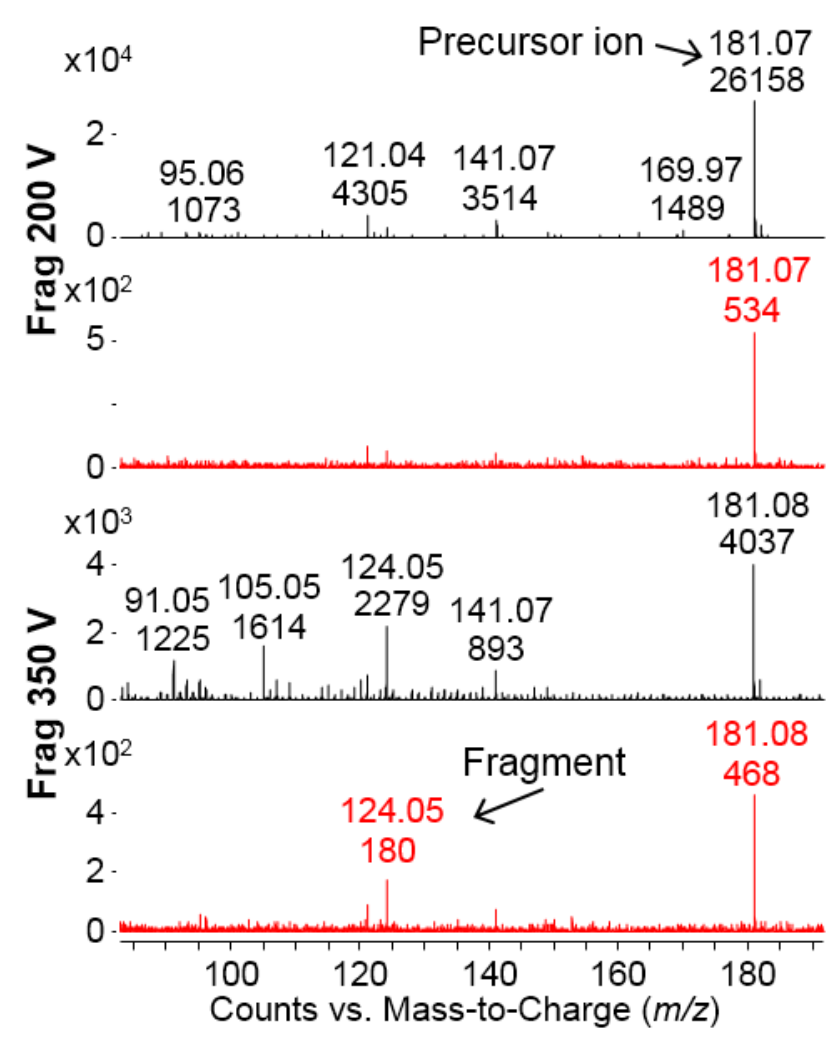

Figure 6: Mass spectra at retention time $2.53 \mathrm{~min}$ from (a) LC-MS (black) and (b) LC-FAIMSMS (red, CF 1.06 Td, DF 240 Td) analysis of urine at fragmentor voltage $200 \mathrm{~V}$ and (c) LCMS and (d) LC-FAIMS-MS at fragmentor voltage $350 \mathrm{~V}$. 
Increasing peak capacity in non-targeted omics applications by combining full scan FAIMS with liquid chromatography-mass spectrometry

Kayleigh L. Arthur, Matthew A. Turner, James C. Reynolds and Colin S. Creaser*

Centre for Analytical Science, Department of Chemistry, Loughborough University, Loughborough, Leicestershire, LE11 3TU, UK

Supplementary information 


\section{Supplementary experimental methods}

Sample preparation for FAIMS-MS and TWIMS-MS analysis

A urine aliquot $(500 \mu \mathrm{L})$ was removed from the $-80^{\circ} \mathrm{C}$ freezer and thawed for 20 mins at room temperature. The aliquot was diluted 1:1 with water and centrifuged at $12200 \mathrm{rpm}$ for 10 mins at ambient temperature. The extract was loaded onto a preconditioned (with ACN (1 mL) and water $(1 \mathrm{~mL})) 200 \mathrm{mg} \mathrm{C18CU}$ solid phase extraction (SPE) cartridge (Caliper Life Sciences, Seer Green, UK), washed with ACN:water (14:86 v/v, $1 \mathrm{~mL}$ ) and eluted with ACN $(3 \mathrm{~mL})$. The SPE eluent was then evaporated to near dryness (using $\mathrm{N}_{2}$ ) and reconstituted in $500 \mu \mathrm{L} \mathrm{ACN}$ :water (98:2 $\mathrm{v} / \mathrm{v}$ ) with $10 \mathrm{mM}$ ammonium acetate and $0.1 \%$ acetic acid.

\section{Instrumentation}

FAIMS-MS analysis was performed on an Agilent 6230 TOF MS (Agilent Technologies, Santa Clara, USA) fitted with a prototype chip-based planar FAIMS device (ultraFAIMS, Owlstone Ltd., Cambridge, UK) in the ESI source region, in front of the inlet capillary. The MS nebulizer pressure was set to 50 psig with a sheath gas $\left(\mathrm{N}_{2}\right)$ flow of $11 \mathrm{~L} \mathrm{~min}^{-1}$ at $250{ }^{\circ} \mathrm{C}$, with a nozzle voltage of $2000 \mathrm{~V}$. The capillary voltage was set to $3500 \mathrm{~V}$, the fragmentor voltage was set to $200 \mathrm{~V}$ and the drying gas $\left(\mathrm{N}_{2}\right)$ flow set to $7 \mathrm{~L} \mathrm{~min}^{-1}$ at $150^{\circ} \mathrm{C}$. The MS scan rate was set to 10 scans s$^{-1}$ in the mass range $m / z$ 80-1500 in positive ion mode. The two-dimensional FAIMS scan (or sweep) was performed for DF 180 to $280 \mathrm{Td}$ (10 Td steps) and CF -2 to $5 \mathrm{Td}$ (0.05 Td steps).

TWIMS-MS was performed on a Synapt G2-S high-resolution MS (Waters Corp., Milford, MA, USA). The MS ESI source was operated in positive ion mode, with capillary voltage set to $2 \mathrm{kV}$ and sampling cone set to $30 \mathrm{~V}$. The source and desolvation temperatures were set to $120^{\circ} \mathrm{C}$ and $300^{\circ} \mathrm{C}$ respectively, gas flow $\left(\mathrm{N}_{2}\right)$ rates were set to $30 \mathrm{~L} / \mathrm{h}$ and $600 \mathrm{~L} / \mathrm{h}$ respectively and the nebulizer pressure was set to 7 bar. The tri-wave drift cell conditions were set to a wave velocity of $300 \mathrm{~m} / \mathrm{s}$ with the wave height ramped from $10 \mathrm{~V}$ to $40 \mathrm{~V}$ (ramped over the full IMS cycle).

Both FAIMS-MS and TWIMS-MS analyses were performed by using a syringe pump for direct infusion into the ESI source, operated at a $10 \mu \mathrm{L} \mathrm{min}{ }^{-1}$ sample infusion rate.

Data files for both FAIMS-MS and TWIMS-MS were exported as CSV files from MassHunter (Agilent Technologies, Santa Clara, USA) and Driftscope (Waters, Manchester, UK) respectively, for importation into OriginLab 2015 (Academic version, b9.2.272). 




Supplementary Figure 1: Schematic diagram of the connections to allow synchronization of the FAIMS scan with the LC and MS, showing the contact closure interface from the binary pump of the LC to the computer to trigger the start of the FAIMS scan. 

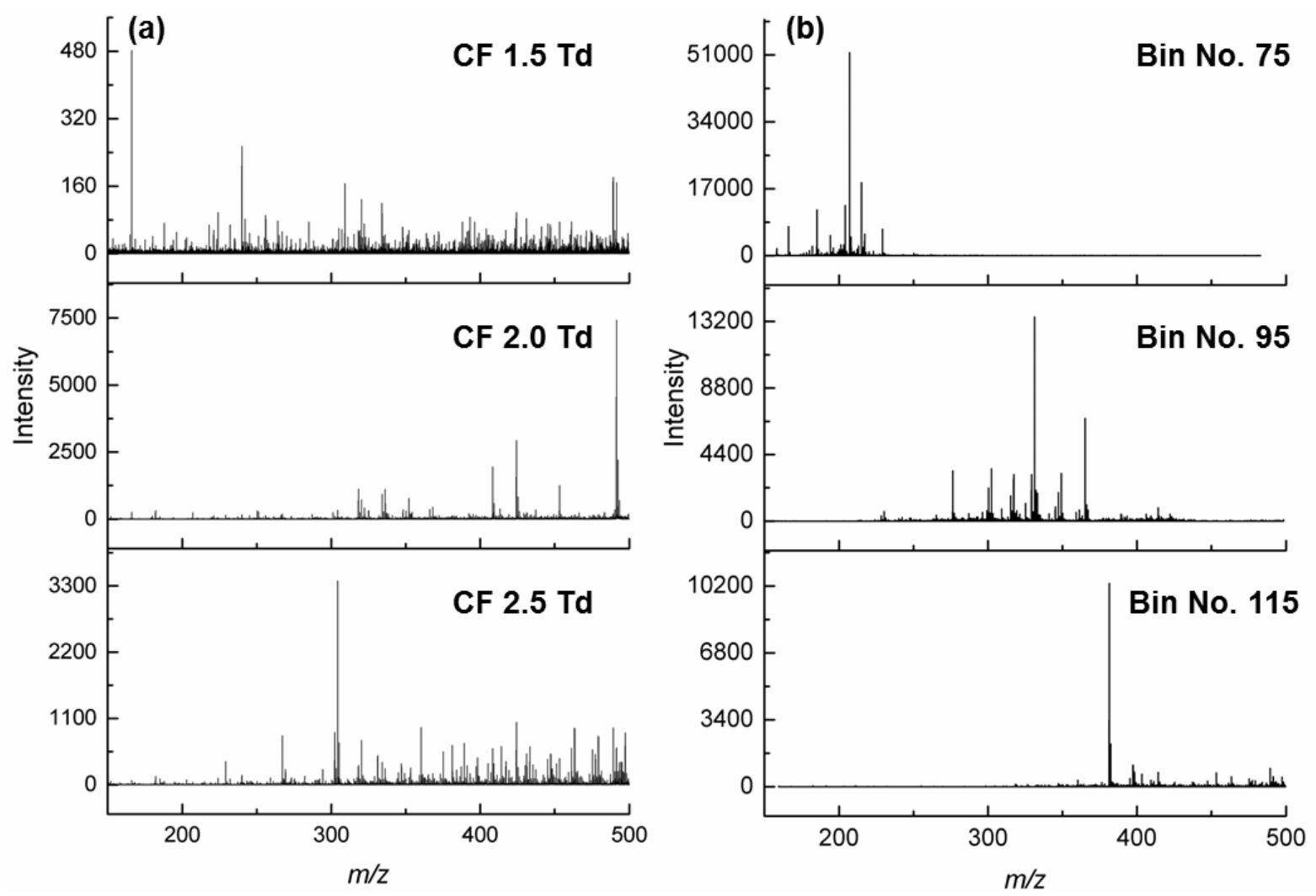

Supplementary Figure 2: Extracted mass spectra from (a) ESI-FAIMS-MS and (b) ESITWIMS-MS of urine at increasing CFs and bin numbers.

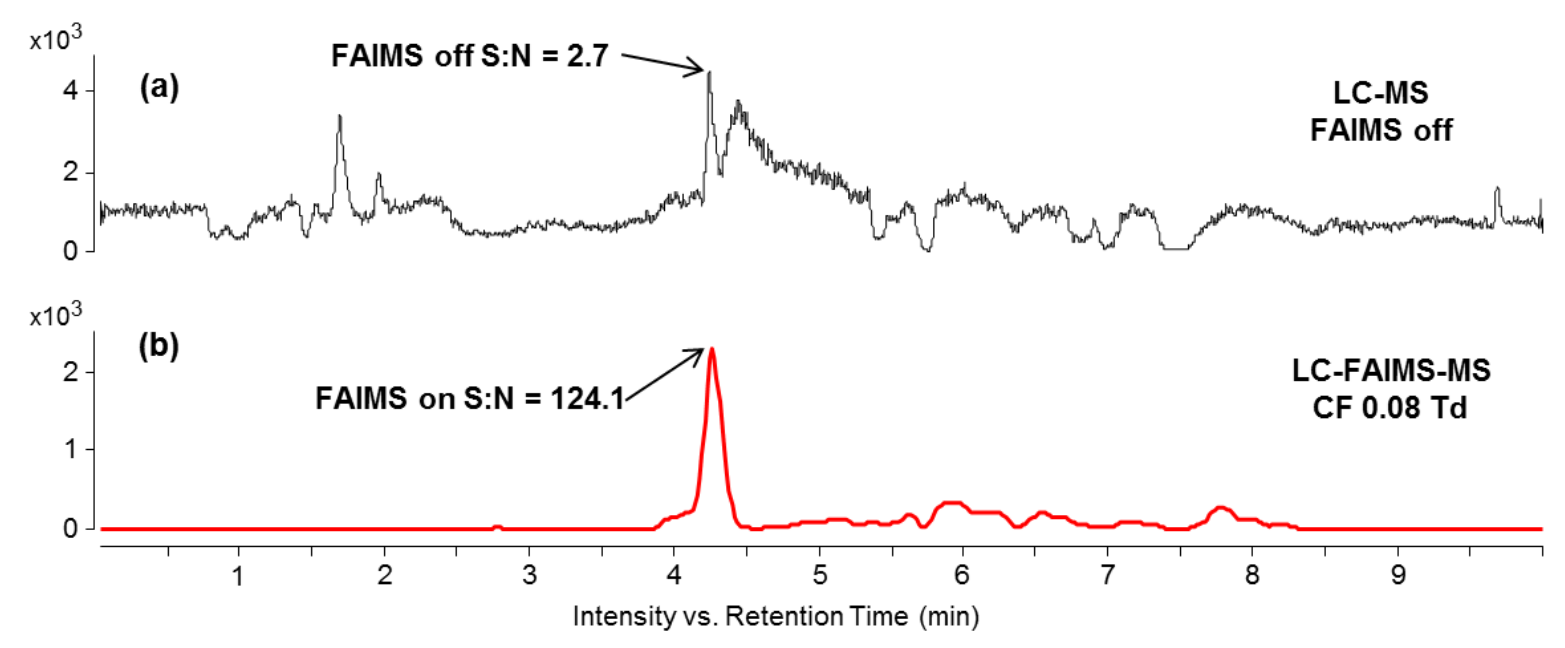

Supplementary Figure 3: Extracted ion chromatograms for $\mathrm{m} / \mathrm{z} 331.21$ showing (a) LC-MS and (b) LC-FAIMS-MS (CF 0.08 Td, DF 240 Td). 

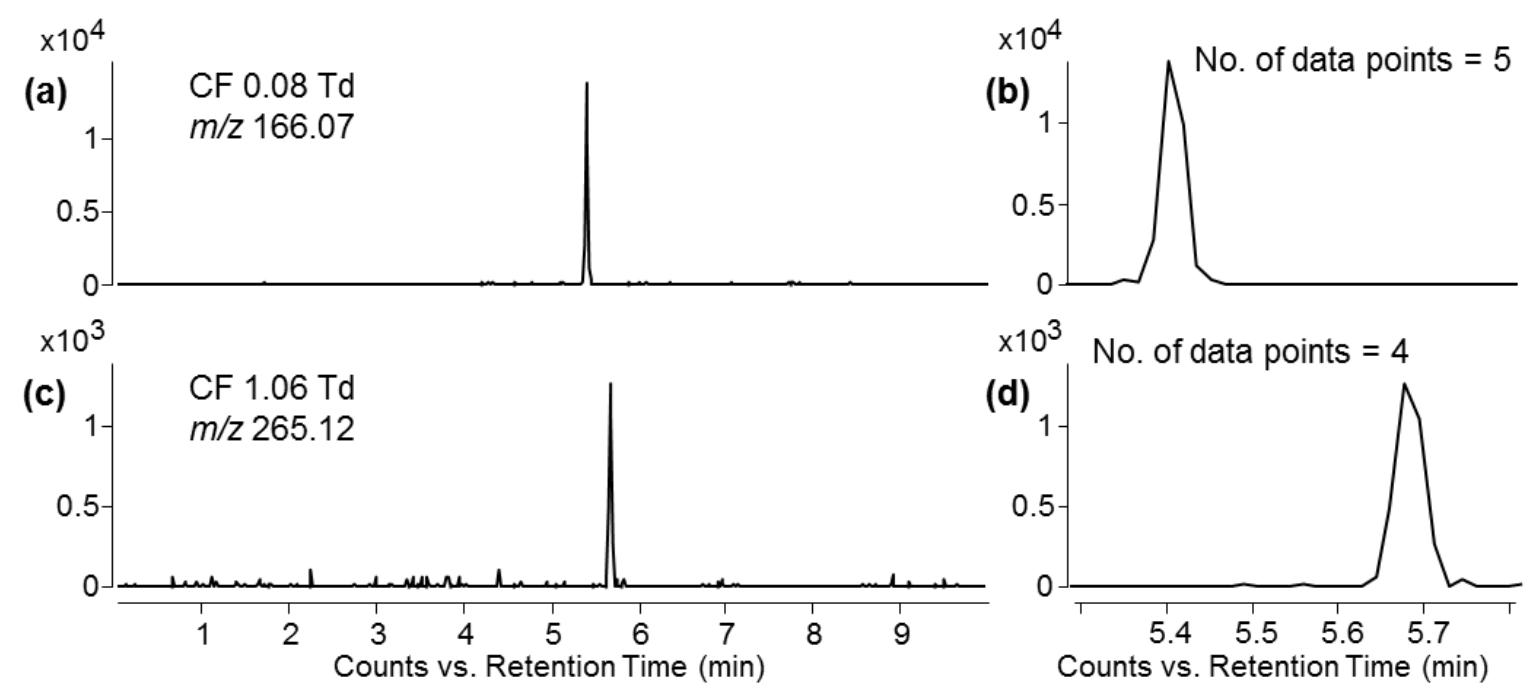

Supplementary Figure 4: Extracted ion chromatograms (at DF $240 \mathrm{Td}$ ) of (a) and (b) $\mathrm{m} / \mathrm{z}$ 166.07 at CF $0.08 \mathrm{Td}$, and (c) and (d) $\mathrm{m} / \mathrm{z} 265.12$ at CF $1.06 \mathrm{Td}$ showing the number of data points across the deconvoluted chromatographic peaks, each extracted from a single CF acquired over the full CF range from the scanning LC-FAIMS-MS data acquisition. 